\title{
Fisher' (1967) Ambiguity Revised: A Catastrophe Theory’ Approach (I)
}

\author{
David Catela, Liliana Gens, Cristiana Mercê \\ Polytechnic Institute of Santarém, Santarém, Portugal
}

\begin{abstract}
Half of a century has passed on Fisher' ambiguity pictures (1967). To celebrate this occasion, we have analysed his data, using the polynomial function for a cusp catastrophe, and we have discussed the results under dynamical systems theory' propositions.
\end{abstract}

Keywords: catastrophe, potential landscape, visual perception

\section{Introduction}

Leeper (1935) observed that in the Boring' (1930) wife/mother-in-law figure, which depicts the face of the younger female (wife) was dominant upon $65 \%$ of occasions. So, Botwinick (1961) prepared three versions of his husband/father-in-law figure, emphasizing the father-in-law aspect in one, the husband aspect in another one, and neither in the third. In this latter figure, 24 subjects of 51 reported the husband aspect. To find a similar probability of appearance of either aspect, in his Man/Girl version, Fisher (1967) also decided to conceive a central figure with both aspects apparently equal in dominance (Picture Number 8 in Figure 1), but more 14 figures, seven for either side of the central one, arranged with a similar incremental difference, till Man and Girl extremes. These 15 figures were shown to 50 subjects in pseudo-random orders and frequencies obtained are shown in Figure 1.

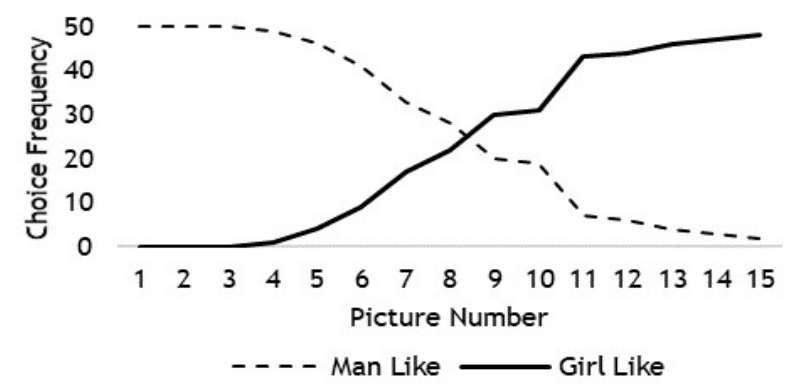

Figure 1. Frequencies obtained by Fisher (1967), for each of 15 pictures, from the one predominantly Man like (Picture Number 1) till the predominantly Girl like (Picture Number 15), with a central (Picture Number 8) with both aspects balanced.

David Catela, Ph.D., Applied Psychology Coordinator (UIIPS), Life Quality Research Center (CIEQV), Polytechnic Institute of Santarém branch, Santarém, Portugal.

Liliana Gens, M.Sc., Motor Development, Polytechnic Institute of Santarém, Santarém, Portugal.

Cristiana Mercê, M.Sc., Motor Development, UIIPS Applied Psychology Member, Polytechnic Institute of Santarém, Santarém, Portugal. 
However, what is particularly interesting for us is not the extreme choices; they confirm a clear figure perception, nor the similar distribution along progressively distal figures, but the frequencies in the central and in the adjacent ones. Why the same picture affords distinct responses? This catastrophic phenomenon is labelled bi-stability because the same stimulation is detected by the perceptual system as two different patterns, i.e., the intermediate pictures can be assumed as Man or Girl. There is a classical explanation where neural fatigue (satiation hypothesis) results in spontaneous perceptual change (Kohler \& Wallach, 1944). Since some transformation of initial conditions is needed to occur such a perceptual change, e.g., the Man like choice endures till Fisher' Picture Number 3, the satiation hypothesis specifies that an initially established percept processes a certain strength that loses as initial conditions evolve. This proposition is very similar to the concept of attractor in dynamical systems models, like catastrophe theory. Subjects feel attracted to see Man or Girl according to the strength (dominant aspects) of these two attractors (Man or Girl). In fact, bi-stable figures seem to be well-described by elementary catastrophe models, like the cusp model, allowing to detect singularities of the occurrence of a catastrophe, like hysteresis (Poston \& Stewart, 1978; Stewart \& Peregoy, 1983; L. K. Ta'edd, O. Ta'eed, \& Wright, 1988) and other inherent flags (Gilmore, 1981).

\section{Method}

Mathematically, one form of the components of the dynamical systems that are simulated is using differential equations. Thus, in analysing these equations, we are in fact analysing all the possible behaviors of the dynamic systems that are described by them. The differential equations use two main ways to express the dynamic system: parameters and variables. The parameters describe the constraints of the systems and are used to express the constant structure of the dynamic system. The variables describe the states of the dynamic system and are used to express aspects of the system that change over time (Abramiuk, 2009). Dynamic system models describe typical system-wide behavior. Thus, behavior is seen as a self-organized pattern that emerges from the interaction between subsystems, called the collective variable. The behavior of the system, as a whole, can be described in terms of dynamics as being of low dimensional order. Differential equations can be used to describe the behavior of a system and polynomial functions capture the long-term behavior of the underlying dynamics. The polynomial function can be interpreted as describing an attractive landscape (potential landscape). Consequently, the transitions between the different modes of behavior can be induced. The parameters that lead the system through these different behavioral patterns are called control parameters that somehow determine (but do not prescribe) the emerging pattern; for this reason, they are referred to as nonspecific control parameters. In a dynamic system, the collective variable is continuously defined, since all possible states remain in a continuous mode, referred to as space state. The regions of the space state are stable, that is, even if the transitions from one qualitative state to another involve a trajectory through the intermediate states, there are a variety of limited states that can be maintained long enough to sustain a certain pattern of behavior (Van Rooij, Bongers, \& Haselager, 2002). In the present study, there are two regions of possible stability: Man like or Girl like.

Tuller, Case, Ding, and Kelso (1994), in a study about identification of one of two words with similar pronunciation, were able to mathematically model the evolution of this type of phenomena through the Equation:

$$
V(x)=k x-1 / 2 x^{2}+1 / 4 x^{4}
$$


$V(x)$ is the collective system variable and represents a polynomial function with two minimums, and $k$ is the control parameter that specifies the direction and degree of slope of the polynomial function $V(x)$. In this model, it is assumed that the state of the system, $x$ changes with time under the influence of the attractive landscape.

\section{Results}

For Fisher' (1967) data, the evolution of the potential landscape is as follows (see Figure 2):
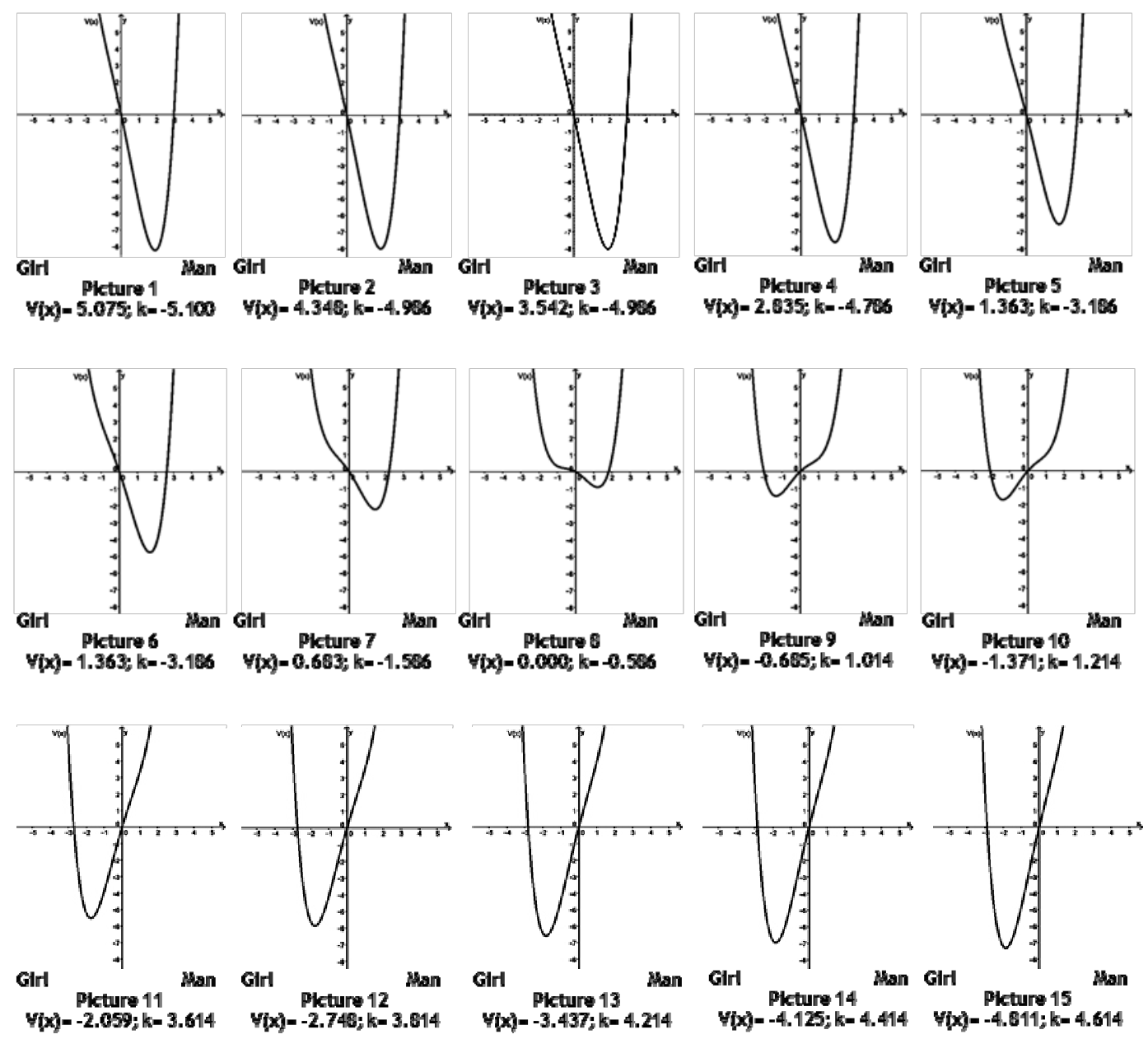

Figure 1. Potential landscapes for pictures 1 to 15 (Source: Fisher, 1967).

As can be observed, in agreement with Fisher' (1967) data, Man like pictures (from 1 to 3) produced a subjects' strong attraction, which is stronger than the three last ones for Girl like evidenced by a deeper valley. This stronger attraction is still evident in the intermediate picture, succeeded by a shy sudden jump and a slower deepening of perception' attraction to Girl like.

\section{Discussion}

Attractors are somewhere in the Gestalt of lines of the pictures, that is, as he said were manipulated in 51 initial ones and progressively selected till the final 15, based on the distribution of the frequency of choices for Man like and Girl like. So, what Fisher left us was a pictorial instrument, not so symmetrical as actual stylised 
computer versions, but probably easier to understand and accept by special populations, like children.

Fisher presented the pictures to subjects in a random order; however, the dynamical theories propose that the initial conditions constrain the evolution of the system. It would be interesting to analyse person's response using methods according catastrophe theory.

\section{References}

Abramiuk, M. A. (2009). Appreciating and embodying the dynamics of large-scale systems: Some examples from the present and the past. Ecological Psychology, 21, 68-95.

Boring, E. G. (1930). A new ambiguous figure. American Journal of Psychology, 42, 444-445.

Botwinick, J. (1961). Husband and father-in-law: A reversible figure. American Journal of Psychology, 74, 312-313.

Fisher, G.H. (1967). Preparation of ambiguous stimulus materials. Perception \& Psychophysics, 2(9), 421-422.

Gilmore, R. (1981). Catastrophe theory for scientists and engineers. New York: Wiley.

Hock, H. S., Schöner, G., \& Voss, A. (1997). The influence of adaptation and stochastic fluctuations on spontaneous perceptual changes for bistable stimuli. Perception \& Psychophysics, 59(4), 509-522.

Kohler, W., \& Wallach, H. (1944). Figural after-effects: An investigation of visual processes. Proceedings of the American Philosophical Society, 88, 269-357.

Leeper, R. W. (1935). A study of a neglected portion of the field of learning: The development of sensory organization. Journal of Genetic Psychology, 46, 41-75.

Poston, T., \& Stewart, I. (1978). Nonlinear modeling of multistable perception. Behavioral Science, 23(4), 318-334.

Stewart, I. N., \& Peregoy, P. L. (1983). Catastrophe theory modelling in psychology. Psychological Bulletin, 94(2), 336-362.

Ta'edd, L. K., Ta'eed, O., \& Wright, J. E. (1988). Determinants involved in the perception of the Necker cube: An application of catastrophe theory. Behavioral Science, 33(2), 97-115.

Tuller, B., Case, P., Ding, M., \& Kelso, J. A. (1994). The nonlinear dynamics of speech categorization. Journal of Experimental Psychology: Human Perception and Performance, 20(1), 3-16.

Van Rooij, I., Bongers, R. M., \& Haselager, F. G. (2002). A non - representational approach to imagined action. Cognitive Science, 26(3), 345-375. 